University of Texas at El Paso

ScholarWorks@UTEP

$3-2020$

\title{
Scale-Invariance and Fuzzy Techniques Explain the Empirical Success of Inverse Distance Weighting and of Dual Inverse Distance Weighting in Geosciences
}

\author{
Laxman Bokati \\ The University of Texas at El Paso, Ibokati@utep.edu \\ Aaron A. Velasco \\ The University of Texas at El Paso, aavelasco@utep.edu \\ Vladik Kreinovich \\ The University of Texas at El Paso, vladik@utep.edu
}

Follow this and additional works at: https://scholarworks.utep.edu/cs_techrep

Part of the Computer Sciences Commons

Comments:

Technical Report: UTEP-CS-20-24

\section{Recommended Citation}

Bokati, Laxman; Velasco, Aaron A.; and Kreinovich, Vladik, "Scale-Invariance and Fuzzy Techniques Explain the Empirical Success of Inverse Distance Weighting and of Dual Inverse Distance Weighting in Geosciences" (2020). Departmental Technical Reports (CS). 1418.

https://scholarworks.utep.edu/cs_techrep/1418

This Article is brought to you for free and open access by the Computer Science at ScholarWorks@UTEP. It has been accepted for inclusion in Departmental Technical Reports (CS) by an authorized administrator of ScholarWorks@UTEP.For more information, please contact Iweber@utep.edu. 


\title{
Scale-Invariance and Fuzzy Techniques Explain the Empirical Success of Inverse Distance Weighting and of Dual Inverse Distance Weighting in Geosciences
}

\author{
Laxman Bokati ${ }^{1}$, Aaron Velasco ${ }^{2}$, and Vladik Kreinovich ${ }^{1,3}$ \\ ${ }^{1}$ Computational Science Program \\ ${ }^{2}$ Department of Geological Sciences \\ ${ }^{3}$ Department of Computer Science \\ University of Texas at El Paso \\ $500 \mathrm{~W}$. University \\ El Paso, Texas 79968, USA \\ lbokati@miners.utep.edu, aavelasco@utep.edu,vladik@utep.edu
}

\begin{abstract}
Once we measure the values of a physical quantity at certain spatial locations, we need to interpolate these values to estimate the value of this quantity at other locations $x$. In geosciences, one of the most widely used interpolation techniques is inverse distance weighting, when we combine the available measurement results with the weights inverse proportional to some power of the distance from $x$ to the measurement location. This empirical formula works well when measurement locations are uniformly distributed, but it leads to biased estimates otherwise. To decrease this bias, researchers recently proposed a more complex dual inverse distance weighting technique. In this paper, we provide a theoretical explanation both for the inverse distance weighting and for the dual inverse distance weighting. Specifically, we show that if we use the general fuzzy ideas to formally describe the desired property of the interpolation procedure, then physically natural scale-invariance requirement select only these two distance weighting techniques.
\end{abstract}

\section{Formulation of the Problem}

Need for interpolation of spatial data. In many practical situations, we are interested in the value of a certain physical quantity at different spatial locations. For example, in geosciences, we may be interested in how elevation and depths of different geological layers depend of the spatial location. In environmental 
sciences, we may be interested in the concentration of different substances in the atmosphere at different locations. etc.

In principle, at each location, we can measure - directly or indirectly the value of the corresponding quantity. However, we can only perform the measurement at a finite number of locations. Since we are interested in the values of the quantity at all possible locations, we need to estimate these values based on the measurement results - i.e., we need to interpolate and extrapolate the spatial data.

In precise terms: we know the values $q_{i}=q\left(x_{i}\right)$ of the quantity of interest $q$ at several locations $x_{i}, i=1,2, \ldots, n$. Based on this information, we would like to estimate the value $q(x)$ of this quantity at a given location $x$.

Inverse distance weighting. A reasonable estimate $q$ for $q(x)$ is a weighted average of the known values $q\left(x_{i}\right): q=\sum_{i=1}^{n} w_{i} \cdot q_{i}$, with $\sum_{i=1}^{n} w_{i}=1$. Naturally, the closer is the point $x$ to the point $x_{i}$, the larger should be the weight $w_{i}$ - and if the distance $d\left(x, x_{i}\right)$ is large, then the value $q\left(x_{i}\right)$ should not affect our estimate at all. So, the weight $w_{i}$ with which we take the value $q_{i}$ should decrease with the distance.

Empirically, it turns out that the best interpolation is attained when we take the weight proportional to some negative power of the distance: $w_{i} \sim$ $\left(d\left(x, x_{i}\right)\right)^{-p}$ for some $p>0$. Since the weights have to add up to 1 , we thus get

$$
w_{i}=\frac{\left(d\left(x, x_{i}\right)\right)^{-p}}{\sum_{j=1}^{n}\left(d\left(x, x_{j}\right)\right)^{-p}} .
$$

This method - known as inverse distance weighting - is one of most widely used spatial interpolation methods; see, e.g., [3, 4, 5, 8, 9, 14].

First challenge: why inverse distance weighting? In general, the fact that some algorithm is empirically the best means that we tried many other algorithms, and this particular algorithm worked better than everything else we tried. In practice, we cannot try all possible algorithms, we can only try finitely many different algorithms. So, in principle, there could be an algorithm that we did not try and that will work better than the one which is currently empirically the best.

To be absolutely sure that the empirically found algorithm is the best, it is thus not enough to perform more testing: we need to have some theoretical explanation of this algorithm's superiority. Because of this, every time we have some empirically best alternative, it is desirable to come up with a theoretical explanation of why this alternative is indeed the best - and if such an explanation cannot be found, maybe it this alternative is actually not the best?

Thus, the empirical success of inverse distance weighting prompts a natural question: is this indeed the best method? This is the first challenge that we will deal with in this paper. 
Limitations of inverse distance weighting. While the inverse distance weighting method is empirically the best among different distance-dependence interpolation techniques, it has limitations; see, e.g., [7].

Specifically, it works well when we have a reasonably uniformly distributed spatial data. The problem is that in many practical cases, we have more measurements in some areas and fewer in others. For example, when we measure meteorological quantities such as temperature, humidity, wind speed, we usually have plenty of sensors (and thus, plenty of measurement results) in cities and other densely populated areas, but much fewer measurements in not so densely populated areas - e.g., in the deserts.

Let us provide a simple example explaining why this may lead to a problem. Suppose that we have two locations $A$ and $B$ at which we perform measurements:

- Location $A$ is densely populated, so we have two measurement results $q_{A}$ and $q_{A^{\prime}}$ from this area.

- Location $B$ is a desert, so we have only one measurement result $q_{B}$ from this location.

Since locations $A$ and $A^{\prime}$ are very close, the corresponding values are also very close, so we can safely assume that they are equal: $q_{A}=q_{A^{\prime}}$. Suppose that we want to use these three measurement results to predict the value of the quantity $x$ at a midpoint $C$ between the locations $A$ and $B$.

Since $C$ is exactly in the middle between $A$ and $B$, when estimating $q_{C}$, intuitively, we should combine the values $q_{A}$ and $q_{B}$ with equal weights, i.e., take $q_{C}=\frac{q_{A}+q_{B}}{2}$. From the commonsense viewpoint, it should not matter whether we made a single measurement at the location $A$ or we made two different measurements.

However, this is not what we get if we apply the inverse distance weighting. Indeed, in this case, since all the distance are equal $d(A, C)=d\left(A^{\prime}, C\right)=$ $d(B, C)$, the inverse distance weighting leads to

$$
q_{C}=\frac{q_{A}+q_{A^{\prime}}+q_{B}}{3}=\frac{2}{3} \cdot q_{A}+\frac{1}{3} \cdot q_{B} .
$$

Dual inverse distance weighting: an empirically efficient way to overcome this limitation. To overcome the above limitation, a recent paper [7] proposed a new method called dual inverse distance weighting, a method that is empirically better than all previously proposed attempts to overcome this limitation.

In this method, instead of simply using the weight $w_{i} \sim\left(d\left(x, x_{i}\right)\right)^{-p}$ depending on the distance, we also give more weight to the points which are more distant from others - and less weight to points which are close to others, by using a formula $w_{i} \sim\left(d\left(x, x_{i}\right)\right)^{-p} \cdot\left(\sum_{j \neq i}\left(d\left(x_{i}, x_{j}\right)\right)^{p_{2}}\right)$, for some $p_{2}>0$. 
Let us show, on an example, that this idea indeed helps overcome the above limitation. Indeed, in the above example of extrapolating from the three points $A \approx A^{\prime}$ and $B$ to the midpoint $C$ between $A$ and $B$ (for which $d(A, C)=$ $d(B, C))$, we have $d\left(A, A^{\prime}\right) \approx 0$ and $d(A, B) \approx d\left(A^{\prime}, B\right)$. Thus, we get the following expressions for the additional factors $f_{i}=\sum_{j \neq i}\left(d\left(x_{i}, x_{j}\right)\right)^{p_{2}}$ :

$$
\begin{gathered}
f_{A}=\left(d\left(A, A^{\prime}\right)\right)^{p_{2}}+(d(A, B))^{p_{2}} \approx(d(A, B))^{p_{2}}, \\
f_{A^{\prime}}=\left(d\left(A^{\prime}, A\right)\right)^{p_{2}}+\left(d\left(A^{\prime}, B\right)\right)^{p_{2}} \approx(d(A, B))^{p_{2}},
\end{gathered}
$$

and

$$
f_{B}=(d(B, A))^{p_{2}}+\left(d\left(B, A^{\prime}\right)\right)^{p_{2}} \approx 2(d(A, B))^{p_{2}} .
$$

So, the weights $w_{A}$ and $w_{A^{\prime}}$ with which we take the values $q_{A}$ and $q_{A^{\prime}}$ are proportional to

$$
w_{A} \approx w_{A^{\prime}} \sim(d(A, C))^{-p} \cdot f_{A} \approx(d(A, C))^{-p} \cdot(d(A, B))^{p_{2}},
$$

while

$$
w_{B} \approx w_{B} \sim(d(B, C))^{-p} \cdot f_{2} \approx(d(A, C))^{-p} \cdot 2(d(A, B))^{p_{2}} .
$$

The weight $w_{B}$ is thus twice larger than the weights $w_{A}$ and $w_{A^{\prime}}: w_{B}=2 w_{A}=$ $2 w_{A^{\prime}}$. Ao the interpolated value of $q_{C}$ is equal to

$$
q_{C}=\frac{w_{A} \cdot q_{A}+w_{A^{\prime}} \cdot q_{A^{\prime}}+w_{B} \cdot q_{B}}{w_{A}+w_{A^{\prime}}+w_{B}}=\frac{w_{A} \cdot q_{A}+w_{A} \cdot q_{A^{\prime}}+2 w_{A} \cdot q_{A}}{w_{A}+w_{A^{\prime}}+2 w_{A}} .
$$

Dividing both numerator and denominator by $2 w_{A}$ and taking into account that $q_{A^{\prime}}=q_{A}$, we conclude that $q_{C}=\frac{q_{A}+q_{B}}{2}$, i.e., exactly the value that we wanted.

Second challenge: why dual inverse distance weighting? In view of the above, it is also desirable to come up with a theoretical explanation for the dual inverse weighting method as well. This is the second challenge that we take on in this paper.

\section{What Is Scale Invariance and How It Ex- plains the Empirical Success of Inverse Dis- tance Weighting}

What is scale invariance. When we process the values of physical quantities, we process real numbers. It is important to take into account, however, that the numerical value of each quantity depends on the measuring unit. For example, suppose that we measure the distance in kilometers and get a numerical value $d$ such as $2 \mathrm{~km}$. Alternatively, we could use meters instead of kilometers. In this case, the exact same distance will be described by a different number: $2000 \mathrm{~m}$. 
In general, if we replace the original measuring unit with a new one which is $\lambda$ times smaller, all numerical values will be multiplied by $\lambda$, i.e., instead of the original numerical value $x$, we will get a new numerical value $\lambda \cdot x$.

Scale-invariance means, in our case, that the result of interpolation should not change if we simply change the measuring unit. Let us analyze how this natural requirement affects interpolation.

General case of distance-dependent interpolation. Let us consider the general case, when the further the point, the smaller the weight, i.e., in precise terms, when the weight $w_{i}$ is proportional to $f\left(d\left(x, x_{i}\right)\right)$ for some decreasing function $f(z): w_{i} \sim f\left(d\left(x, x_{i}\right)\right)$. Since the weights should add up to 1 , we conclude that

$$
w_{i}=\frac{f\left(d\left(x, x_{i}\right)\right)}{\sum_{j} f\left(d\left(x, x_{j}\right)\right)},
$$

and thus, our estimate $q$ for $q(x)$ should take the form

$$
q=\sum_{i=1}^{n} \frac{f\left(d\left(x, x_{i}\right)\right)}{\sum_{j} f\left(d\left(x, x_{j}\right)\right)} \cdot q_{i} .
$$

In this case, scale-invariance means that for each $\lambda>0$, if we replace all the numerical distance values $d\left(x, x_{i}\right)$ with "re-scaled' values $\lambda \cdot d\left(x, x_{i}\right)$, then we should get the exact same interpolation result, i.e., that for all possible values of $q_{i}$ and $d\left(x, x_{i}\right)$, we should have

$$
\sum_{i=1}^{n} \frac{f\left(\lambda \cdot d\left(x, x_{i}\right)\right)}{\sum_{j} f\left(\lambda \cdot d\left(x, x_{j}\right)\right)} \cdot q_{i}=\sum_{i=1}^{n} \frac{f\left(d\left(x, x_{i}\right)\right)}{\sum_{j} f\left(d\left(x, x_{j}\right)\right)} \cdot q_{i} .
$$

Scale-invariance leads to inverse distance scaling. Let us show that the requirement (3) indeed leads to inverse distance scaling.

Indeed, let us consider the case when we have only two measurement results:

- at the point $x_{1}$, we got the value $q_{1}=1$, and

- at point $x_{2}$, we got the value $q_{2}=0$.

Then, for any point $x$, if we use the original distance values $d_{1} \stackrel{\text { def }}{=} d\left(x, x_{1}\right)$ and $d_{2} \stackrel{\text { def }}{=} d\left(x, x_{2}\right)$, the interpolated value $q$ at this point will have the form

$$
q=\frac{f\left(d_{1}\right)}{f\left(d_{1}\right)+f\left(d_{2}\right)}
$$

On the other hand, if we use a $\lambda$ times smaller measuring unit, then the extrapolation formula leads to the values

$$
\frac{f\left(\lambda \cdot d_{1}\right)}{f\left(\lambda \cdot d_{1}\right)+f\left(\lambda \cdot d_{2}\right)} .
$$


The requirement that the interpolation value does not change if we simply change the measuring unit implies that these two expression must coincide, i.e., that we must have:

$$
\frac{f\left(\lambda \cdot d_{1}\right)}{f\left(\lambda \cdot d_{1}\right)+f\left(\lambda \cdot d_{2}\right)}=\frac{f\left(d_{1}\right)}{f\left(d_{1}\right)+f\left(d_{2}\right)} .
$$

If we take the inverse of both sides of this formula, i.e., flip the numerator and denominator in both sides, we get

$$
\frac{f\left(\lambda \cdot d_{1}\right)+f\left(\lambda \cdot d_{2}\right)}{f\left(\lambda \cdot d_{1}\right)}=\frac{f\left(d_{1}\right)+f\left(d_{2}\right)}{f\left(d_{1}\right)} .
$$

Subtracting number 1 from both sides, we get a simplified expression

$$
\frac{f\left(\lambda \cdot d_{2}\right)}{f\left(\lambda \cdot d_{1}\right)}=\frac{f\left(d_{2}\right)}{f\left(d_{1}\right)} .
$$

If we divide both sides by $f\left(d_{2}\right)$ and multiply by $f\left(\lambda \cdot d_{1}\right)$, we get the equivalent equality in which variables $d_{1}$ and $d_{2}$ are separated:

$$
\frac{f\left(\lambda \cdot d_{2}\right)}{f\left(d_{2}\right)}=\frac{f\left(\lambda \cdot d_{1}\right)}{f\left(d_{1}\right)} .
$$

The left-hand side of this formula does not depend on $d_{1}$; thus, the right-hand side does not depend on $d_{1}$ either, it must thus depend only on $\lambda$. Let us denote this right-hand side by $c(\lambda)$. Then, from $\frac{f\left(\lambda \cdot d_{1}\right)}{f\left(d_{1}\right)}=c(\lambda)$, we conclude that

$$
f\left(\lambda \cdot d_{1}\right)=c(\lambda) \cdot f\left(d_{1}\right)
$$

for all possible values of $\lambda>0$ and $d_{1}$.

It is known that for decreasing functions $f(z)$, the only solutions to the functional equation (8) are functions $f(z)=c \cdot z^{-p}$ for some $p>0$; see, e.g., [1]. For this function $f(z)$, the extrapolated value has the form $\sum f_{i} \cdot q_{i}$, with

$$
f_{i}=\frac{c \cdot\left(d\left(x, x_{i}\right)\right)^{-p}}{\sum_{j=1}^{n} c \cdot\left(d\left(x, x_{j}\right)\right)^{-p}}
$$

If we divide both numerator and denominator by $c$, we get exactly the inverse distance weighting formula.

Thus, scale-invariance indeed leads to inverse distance weighting.

Comment. For smooth function $f(x)$, the above result about solutions of the functional equation can be easily derived. Indeed, differentiating both sides of the equality (8) by $\lambda$ and taking $\lambda=1$, we get

$$
f^{\prime}\left(d_{1}\right) \cdot d_{1}=\alpha \cdot f\left(d_{1}\right),
$$


where we denoted $\alpha \stackrel{\text { def }}{=} c^{\prime}(1)$, i.e., we have

$$
\frac{d f}{d d_{1}}=\alpha \cdot f
$$

If we divide both sides by $f$ and multiply by $d d_{1}$, we separate $d_{1}$ and $f: \frac{d f}{f}=$ $\alpha \cdot \frac{d d_{1}}{d_{1}}$. Integrating both sides, we get $\ln (f)=\alpha \cdot \ln \left(d_{1}\right)+C$, where $C$ is the integration constant. Applying $\exp (z)$ to both sides and taking into account that $\exp (\ln (f))=f$ and

$\exp \left(\alpha \cdot \ln \left(d_{1}\right)+C\right)=\exp \left(\alpha \cdot \ln \left(d_{1}\right)\right) \cdot \exp (C)=\exp (C) \cdot\left(\exp \left(\ln \left(d_{1}\right)\right)^{\alpha}=\exp (C) \cdot d_{1}^{\alpha}\right.$,

we get $f\left(d_{1}\right)=c \cdot d_{1}^{\alpha}$, where we denoted $c \stackrel{\text { def }}{=} \exp (C)$. Since the function $f(z)$ is decreasing, we should have $\alpha<0$, i.e., $\alpha=-p$ for some $p>0$. The statement is proven.

\section{Scale Invariance and Fuzzy Techniques Ex- plain Dual Inverse Weighting}

What we want: informal description. In the previous section, when computing the estimate $q$ for the value $q(x)$ of the desired quantity at a location $x$, we used, in effect, the weighted average of the measurements results $q_{i}$, with the weights decreasing as the distance $d\left(x, x_{i}\right)$ decreases - i.e., in more precise terms, with weights proportional to $f\left(d\left(x, x_{i}\right)\right)$ for some decreasing function $f(z)$. In this case, scale-invariance implies that $f(z)=z^{-p}$ for some $p>0$.

As we have mentioned in Section 1, we need to also give more weight to measurements at locations $x_{i}$ which are far away from other location - and, correspondingly, less weight to measurements at locations which are close to other locations. In terms of weights, we would like to multiply the previous weights $f\left(d\left(x, x_{i}\right)\right)=\left(d\left(x, x_{i}\right)\right)^{-p}$ by an additional factor $f_{i}$ depending on how far away is location $x_{i}$ from other locations. The further away the location $x_{i}$ from other locations, the higher the factor $f_{i}$ shall be. In other words, the factor $f_{i}$ should be larger or smaller depending on our degree of confidence in the following statement:

$$
d\left(x_{i}, x_{1}\right) \text { is large and } d\left(x_{i}, x_{2}\right) \text { is large and } \ldots d\left(x_{i}, x_{n}\right) \text { is large. }
$$

Let us use fuzzy techniques to translate this informal statements into precise terms. To translate the above informal statement into precise terms, a reasonable idea is to use fuzzy techniques - techniques specifically designed for such a translation; see, e.g., $[2,6,10,12,13,15]$. In this technique, to each basic statement - like " $d$ is large" - we assign a degree to which, according to the expert, this statement is true. This degree is usually denoted by $\mu(d)$. In terms of these notations: 
- the degree to which $d\left(x_{i}, x_{1}\right)$ is large is equal to $\mu\left(d\left(x_{i}, x_{1}\right)\right)$;

- the degree to which $d\left(x_{i}, x_{2}\right)$ is large is equal to $\mu\left(d\left(x_{i}, x_{2}\right)\right)$; etc.

To estimate the degree to which the above "and"-statement is satisfied, fuzzy techniques suggest that we combine the above degrees by using an appropriate "and"-operation (=t-norm) $\left.f_{\&}(a, b)\right)$. Thus, we get the following degree:

$f_{\&}\left(\mu\left(d\left(x_{i}, x_{1}\right)\right), \mu\left(d\left(x_{i}, x_{2}\right)\right), \ldots, \mu\left(d\left(x_{i}, x_{i-1}\right)\right), \mu\left(d\left(x_{i}, x_{i+1}\right)\right), \ldots, \mu\left(d\left(x_{i}, x_{n}\right)\right)\right)$.

It is known - see, e.g., [11] - that for any "and"-operation and for any $\varepsilon>0$, there exists an $\varepsilon$-close "and"-operation of the type $f_{\&}(a, b)=g^{-1}(g(a)+g(b))$ for some monotonic function $g(a)$, where $g^{-1}(a)$ denotes the inverse function (i.e., the function for which $g^{-1}(a)=b$ if and only if $g(b)=a$ ). Since the approximation error $\varepsilon$ can be arbitrarily small, for all practical purposes, we can safely assume that the actual "and"-operation has this $g$-based form. Substituting this expression for the "and"-operation into the above formula, we conclude that $f_{i}$ should monotonically depend on the expression

$$
g^{-1}\left(g\left(\mu\left(d\left(x_{i}, x_{1}\right)\right)\right)+\ldots+g\left(\mu\left(d\left(x_{i}, x_{n}\right)\right)\right)\right) .
$$

Since the function $g^{-1}$ is monotonic, this means that $f_{i}$ is a monotonic function of the expression

$$
\left.G\left(d\left(x_{i}, x_{1}\right)\right)+\ldots+G\left(d\left(x_{i}, x_{n}\right)\right)\right),
$$

where we denoted $G(d) \stackrel{\text { def }}{=} g(\mu(d))$. In other words, we conclude that

$$
f_{i}=F\left(G\left(d\left(x_{i}, x_{1}\right)\right)+\ldots+G\left(d\left(x_{i}, x_{n}\right)\right)\right)
$$

for some monotonic function $F(z)$.

So, we get an estimate

$$
q=\sum_{i=1}^{n} \frac{f_{i} \cdot\left(d\left(x, x_{i}\right)\right)^{-p} \cdot q_{i}}{\sum_{j=1}^{n} f_{j} \cdot\left(d\left(x, x_{j}\right)\right)^{-p}},
$$

where the factors $f_{i}$ are described by the formula (9).

Let us recall the motivation for the factors $f_{i}$. As we have mentioned earlier, the main motivation for introducing the factors $f_{i}$ is to make sure that for the midpoint $C$ between $A$ and $B$, we will have the estimate $\frac{q_{A}+q_{B}}{2}$, even if we perform two (or more) measurements at the point $A$. Let us analyze for which functions $F(z)$ and $G(z)$ this requirement is satisfied.

For the purpose of this analysis, let us consider the case when we have $m$ measurement locations $A_{1}, \ldots, A_{m}$ in the close vicinity of the location $A$ and one measurement result at location $B$. Let $d$ denote the distance $d(A, B)$ between the locations $A$ and $B$. For all the measurement locations $A_{1}, \ldots, A_{m}$, and $B$, the distance to the point $C$ is the same - equal to $d / 2$. Thus, in this case, 
the factors $\left(d\left(x, x_{i}\right)\right)^{-p}$ in the formula (10) are all equal to each other. So, we can divide both the numerator and the denominator by the formula (10) by this common factor, and get a simplified expression

$$
q=\sum_{i=1}^{n} \frac{f_{i} \cdot q_{i}}{\sum_{j=1}^{n} f_{j}}
$$

Since for the points $A_{1}, \ldots, A_{m}$ we have the same measurement results $q_{i}$ (we will denote them by $q_{A}$ ), and the same factors $f_{i}$ (we will denote them by $f_{A}$ ), we get

$$
q=\frac{m \cdot f_{A} \cdot q_{A}+f_{B} \cdot q_{B}}{m \cdot f_{A}+f_{B}} .
$$

We want to make sure that this value is equal to the arithmetic average $\frac{q_{A}+q_{B}}{2}$. Thus, the coefficient at $q_{A}$ in the formula (11) should be equal to $1 / 2$ :

$$
\frac{m \cdot f_{A}}{m \cdot f_{A}+f_{B}}=\frac{1}{2} .
$$

If we multiply both side by their denominators and subtract $m \cdot f_{A}$ from both sides, we get $m \cdot f_{A}=f_{B}$. Due to the formula (9), this means

$$
m \cdot F(G(d)+(m-1) \cdot G(0))=F(m \cdot G(d)) .
$$

In the limit $d=0$, this formula becomes $m \cdot F(m \cdot G(0))=F(m \cdot G(0))$, thus $F(m \cdot G(0))=0$. Since the function $F(z)$ is monotonic, we cannot have $G(0) \neq 0$, since then we would have $F(z)=0$ for all $z$. Thus, $G(0)=0, F(G(0))=F(0)=$ 0 , and the formula (12) takes the form $F(m \cdot G(d))=m \cdot F(G(d))$. This is true for any value $z=G(d)$, so we have $F(m \cdot z)=m \cdot F(z)$ for all $m$ and $z$.

- In particular, for $z=1$, we get $F(m)=c \cdot m$, where $c \stackrel{\text { def }}{=} F(1)$.

- For $z=1 / m$, we then have $F(1)=c=m \cdot F(1 / m)$, hence

$$
F(1 / m)=c \cdot(1 / m)
$$

- Similarly, we get $F(p / q)=F(p \cdot(1 / q))=p \cdot F(1 / q)=p \cdot(c \cdot(1 / q))=c \cdot(p / q)$. So, for all rational values $z=p / q$, we get $F(z)=c \cdot z$.

Since the function $F(z)$ is monotonic, the formula $F(z)=c \cdot z$ is true for all values $z$.

Dividing both the numerator and the denominator by the coefficient $c$, we conclude that

$$
q=\sum_{i=1}^{n} \frac{F_{i} \cdot\left(d\left(x, x_{i}\right)\right)^{-p} \cdot q_{i}}{\sum_{j=1}^{n} F_{j} \cdot\left(d\left(x, x_{j}\right)\right)^{-p}}
$$


where we denoted

$$
F_{i} \stackrel{\text { def }}{=} G\left(d\left(x_{i}, x_{1}\right)\right)+\ldots+G\left(d\left(x_{i}, x_{n}\right)\right) .
$$

Let us now use scale-invariance. We want to make sure that the estimate (13) does not change after re-scaling $d(x, y) \rightarrow d^{\prime}(x, y)=\lambda \cdot d(x, y)$, i.e., that the same value $q$ should be also equal to

$$
q=\sum_{i=1}^{n} \frac{F_{i}^{\prime} \cdot\left(d^{\prime}\left(x, x_{i}\right)\right)^{-p} \cdot q_{i}}{\sum_{j=1}^{n} F_{j}^{\prime} \cdot\left(d^{\prime}\left(x, x_{j}\right)\right)^{-p}},
$$

where

$$
F_{i}^{\prime}=G\left(d^{\prime}\left(x_{i}, x_{1}\right)\right)+\ldots+G\left(d^{\prime}\left(x_{i}, x_{n}\right)\right) .
$$

Here, $\left(d^{\prime}\left(x, x_{i}\right)\right)^{-p}=\lambda^{-p} \cdot\left(d\left(x, x_{i}\right)\right)^{-p}$. Dividing both the numerator and the denominator of the right-hand side of the formula (15) by $\lambda^{-p}$, we get a simplified expression

$$
q=\sum_{i=1}^{n} \frac{F_{i}^{\prime} \cdot\left(d\left(x, x_{i}\right)\right)^{-p} \cdot q_{i}}{\sum_{j=1}^{n} F_{j}^{\prime} \cdot\left(d\left(x, x_{j}\right)\right)^{-p}} .
$$

The two expressions (13) and (17) are linear in $q_{i}$. Thus, their equality implies that coefficients at each $q_{i}$ must be the same. In particular, this means that the ratios of the coefficients at $q_{1}$ and $q_{2}$ must be equal, i.e., we must have

$$
\frac{F_{1} \cdot\left(d\left(x, x_{1}\right)\right)^{-p}}{F_{2} \cdot\left(d\left(x, x_{2}\right)\right)^{-p}}=\frac{F_{1}^{\prime} \cdot\left(d\left(x, x_{1}\right)\right)^{-p}}{F_{2}^{\prime} \cdot\left(d\left(x, x_{2}\right)\right)^{-p}},
$$

i.e.,

$$
\frac{F_{1}}{F_{2}}=\frac{F_{1}^{\prime}}{F_{2}^{\prime}} .
$$

For the case when we have three points with $d\left(x_{1}, x_{2}\right)=d\left(x_{1}, x_{3}\right)=d$ and $d\left(x_{2}, x_{3}\right)=D$, due to the formula (14), this means that

$$
\frac{2 G(d))}{G(d)+G(D)}=\frac{2 G(\lambda \cdot d))}{G(\lambda \cdot d)+G(\lambda \cdot D)} .
$$

Inverting both sides, multiplying both sides by 2 and subtracting 1 from both sides, we conclude that

$$
\frac{G(D)}{G(d)}=\frac{G(\lambda \cdot D)}{G(\lambda \cdot d)}
$$

for all $\lambda, d$, and $D$. We already know - from the first proof - that this implies that $G(d)=c \cdot d^{p_{2}}$ for some $c$ and $p_{2}$, and that, by deleting both numerator and denominator by $c$, we can get $c=1$.

Thus, we indeed get a justification for the dual inverse distance weighting. 


\section{Acknowledgments}

This work was supported in part by the National Science Foundation grants 1623190 (A Model of Change for Preparing a New Generation for Professional Practice in Computer Science) and HRD-1242122 (Cyber-ShARE Center of Excellence).

\section{References}

[1] J. Aczel and J. Dhombres, Functional Equations in Several Variables, Cambridge University Press, Cambridge, UK, 1989.

[2] R. Belohlavek, J. W. Dauben, and G. J. Klir, Fuzzy Logic and Mathematics: A Historical Perspective, Oxford University Press, New York, 2017.

[3] Q. Chen, G. Liu, X. Ma, G. Marietoz, Z. He, Y. Tian, and Z. Weng, "Local curvature entropy-based 3D terrain representation using a comprehensive quadtree", ISPRS Journal of Photogrammetry and Remote Sensing, 2018, Vol. 139, pp. 130-145.

[4] K. C. Clarke, Analytical and Computer Cartography, Pnetice Hall, Englewood Cliffs, New Jersey, 1990.

[5] N. Henderson and L. Pena, "The inverse distance weighting interpolation applied to a particular form of the path rubes method: theory and computation for advection in uncompressible flow", Applied Mathematics and Computation, 2017, Vol. 304, pp. 114-135.

[6] G. Klir and B. Yuan, Fuzzy Sets and Fuzzy Logic, Prentice Hall, Upper Saddle River, New Jersey, 1995.

[7] Z. Li, X. Zhang, R. Zhu, Z. Zhiang, and Z. Weng, "Integrating data-to-data correlation into inverse distance weighting", Computational Geosciences, 2019, https://doi.org/10.1007/s10596-019-09913-9

[8] Q. Liang, S. Nittel, J. C. Whittier, and S. Bruin, "Real-time inverse distance weighting interpolation for streaming sensor data", Transactions on GIS, 2018, Vol. 22, No. 5, pp. 1179-1204.

[9] I. Loghmari, Y. Timoumi, and A. Messadi, "Performance comparison of two global solar radiation models for spatial interpolation purposes", Renewable and Sustainable Energy Review, 2018, Vol. 82, pp. 837-844.

[10] J. M. Mendel, Uncertain Rule-Based Fuzzy Systems: Introduction and New Directions, Springer, Cham, Switzerland, 2017.

[11] H. T. Nguyen, V. Kreinovich, and P. Wojciechowski, "Strict Archimedean t-norms and t-conorms as universal approximators", International Journal of Approximate Reasoning, 1998, Vol. 18, Nos. 3-4, pp. 239-249. 
[12] H. T. Nguyen, C. L. Walker, and E. A. Walker, A First Course in Fuzzy Logic, Chapman and Hall/CRC, Boca Raton, Florida, 2019.

[13] V. Novák, I. Perfilieva, and J. Močkoř, Mathematical Principles of Fuzzy Logic, Kluwer, Boston, Dordrecht, 1999.

[14] D. Shepard, "A two-dimensional interpolation function for irregularlyspaced data", Proceedings of the 1968 23rd ACM National Conference, 1968 , pp. $517-524$.

[15] L. A. Zadeh, "Fuzzy sets", Information and Control, 1965, Vol. 8, pp. 338353. 